\title{
A BASIC ANALOGUE OF MACROBERT'S E-FUNCTION
}

\author{
by R. P. AGARWAL
}

(Received 10 December, 1959)

1. Introduction and notation. MacRobert [2] in 1937 defined the $E$-function as

$$
E(\alpha, \beta:: z)=\sum_{\alpha, \beta} \Gamma(\alpha) \Gamma(\beta-\alpha) z^{\alpha} F(\alpha ; \alpha-\beta+1 ; z),
$$

where the symbol $\sum_{\alpha, \beta}$ denotes that to the expression following it, a similar expression with $\alpha$ and $\beta$ interchanged is to be added. For (1) he also gave the integral representation

$$
E(\alpha, \beta:: z)=\Gamma(\alpha) \int_{0}^{\infty} e^{-\lambda} \lambda^{\beta-1}(1+\lambda / z)^{-\alpha} d \lambda,
$$

where $\operatorname{Re} \beta>0,|\arg z|<\pi$.

Since 1937 the $E$-function has been generalized and studied in detail by MacRobert and others. In this paper, I give a basic analogue of (1) and study some of its fundamental properties.

The following notation is used throughout the paper. Let

$$
|q|<1, \log q=-w=-\left(w_{1}+i w_{2}\right),
$$

where $w, w_{1}, w_{2}$ are constants, $w_{1}$ and $w_{2}$ being real. Also, let

$$
\begin{aligned}
& \left(q^{a}\right)_{n} \equiv(a)_{n}=\left(1-q^{a}\right)\left(1-q^{a+1}\right) \ldots\left(1-q^{a+n-1}\right), \\
& \left(q^{a}\right)_{0}=1, \quad\left(q^{a}\right)_{-n}=(-)^{n} q^{\frac{1}{2 n(n+1)}} q^{-n a} /\left(q^{1-a}\right)_{n} ;
\end{aligned}
$$

then we define the generalized basic hypergeometric function as

$$
{ }_{r+1} \Phi_{r}\left[\begin{array}{l}
\left.a_{1}, a_{2}, \ldots, a_{r+1} ; z\right] \\
b_{1}, b_{2}, \ldots, b_{r}
\end{array}\right]=\sum_{n=0}^{\infty} \frac{\left(a_{1}\right)_{n} \ldots\left(a_{r+1}\right)_{n} z^{n}}{(q)_{n}\left(b_{1}\right)_{n} \ldots\left(b_{r}\right)_{n}}(|z|<1,
$$

and the "confluent" hypergeometric function as

Also

$$
{ }_{1} \Phi_{1}(a ; b ; z)=\sum_{n=0}^{\infty} \frac{(a)_{n}}{(q)_{n}(b)_{n}} z^{n} q^{\frac{1}{2 n(n-1)}}
$$

Also

$$
\begin{gathered}
E_{q}(x)=\prod_{n=0}^{\infty}\left(1-x q^{n}\right)=\sum_{n=0}^{\infty} \frac{(-)^{n} q^{\frac{1}{n(n-1)}}}{(q)_{n}} x^{n}, \\
(x+y)_{\alpha}=x^{\alpha}\left(1+y x^{-1}\right)_{\alpha}=\prod_{n=0}^{\infty} \frac{\left(1+y x^{-1} q^{n}\right)}{\left(1+y x^{-1} q^{\alpha+n}\right)}, \\
\frac{1}{(1+x)_{\alpha}}={ }_{1} \Phi_{0}(\alpha ;-x), \text { for }|x|<1,
\end{gathered}
$$

and

$$
G(\alpha)=\left\{\prod_{n=0}^{\infty}\left(1-q^{\alpha+n}\right)\right\}^{-1}
$$


Further, following Hahn [1], the basic integral of a function under suitable conditions is defined as

$$
\begin{aligned}
& \underset{0}{x} f(y) d(q y)=x(1-q) \sum_{i=0}^{\infty} q^{i} f\left(q^{i} x\right) \\
& {\underset{x}{x}}_{\infty}^{\infty} f(y) d(q y)=x(1-q) \sum_{j=1}^{\infty} q^{-j} f\left(q^{-j} x\right)
\end{aligned}
$$

and thus

$$
{\underset{0}{0}}_{0}^{\infty} f(y) d(q y)=(1-q) \sum_{j=-\infty}^{\infty} q^{j} f\left(q^{j}\right)
$$

As above we denote the basic integrals by the symbol $\underset{a}{\operatorname{S}} d(q u)$.

2. Definitions. We define the basic analogue of the $E$-function by the integral

$$
E_{q}(\alpha, \beta:: z) \equiv \frac{G(\alpha)}{1-q} \operatorname{S}_{0}^{1} E_{q}(q \lambda) \lambda^{\beta-1} \Phi_{0}(\alpha ;-\lambda / z) d(q \lambda),
$$

where $\operatorname{Re} \beta>0$, and let $\arg \lambda=0$, for simplicity.

We now proceed to evaluate the integral on the right of (3). We know that $\dagger$

$$
\frac{G(\alpha)}{G(1)}{ }_{1} \Phi_{0}(\alpha ;-\lambda / z)=\frac{1}{2 \pi i} \int_{C} \frac{G(\alpha-s) \pi(z / \lambda)^{s}}{G(1-s) \sin \pi s} d s,
$$

where the contour $C$ is a line parallel to $\operatorname{Re}(w s)=0$ with loops, if necessary, to include the poles of $G(\alpha-s)$. The integral converges if $\operatorname{Re}[s \log (z \mid \lambda)-\log \sin \pi s]<0$ for large values of $|s|$ on the contour, i.e. if $\left|\left\{\arg z-w_{1}^{-1} w_{2} \log |z|\right\}\right|<\pi$, since $0<\lambda<1$. Hence (3) gives

$$
E_{q}(\alpha, \beta:: z)=\frac{1}{2 \pi i} \frac{G(1)}{1-q} \operatorname{S}_{0}^{1} E_{q}(q \lambda) \lambda^{\beta-1} d(q \lambda) \int_{C} \frac{G(\alpha-s)}{G(1-s)} \frac{\pi(z / \lambda)^{s}}{\sin \pi s} d s .
$$

Changing the order of integration, which is justified for $\operatorname{Re}(\beta-s)>0$ and the above argument of $z$, we get

$$
\begin{aligned}
E_{q}(\alpha, \beta:: z)= & \frac{1}{2 \pi i} \frac{G(1)}{1-q} \int_{C} \frac{G(\alpha-s)}{G(1-s)} \frac{\pi z^{s}}{\sin \pi s} d s{\underset{0}{1}}_{0}^{1} E_{q}(q \lambda) \lambda^{\beta-s-1} d(q \lambda) \\
& =\frac{1}{2 \pi i} \int_{C} \frac{G(\alpha-s) G(\beta-s)}{G(1-s)} \frac{\pi z^{s}}{\sin \pi s} d s,
\end{aligned}
$$

valid by analytic continuation when $\operatorname{Re} \beta>0$ and $\left|\left\{\arg z-w_{2} w_{1}{ }^{-1} \log |z|\right\}\right|<\pi$.

The contour integral (5) gives another integral representation for the $E_{q}$-function. Evaluating (5) by considering the residues at the poles of $G(\alpha-s)$ and $G(\beta-s)$ [3], we get

$$
E_{q}(\alpha, \beta:: z)=\sum_{\alpha, \beta} \frac{G(\alpha) G(\beta-\alpha)}{G(1)} \prod_{n=0}^{\infty} \frac{\left(1+z^{-1} q^{\alpha+n}\right)\left(1+z q^{1-\alpha+n}\right)}{\left(1+z^{-1} q^{n}\right)\left(1+z q^{1+n}\right)}{ }_{1} \Phi_{1}\left(\alpha ; \alpha-\beta+1 ; z q^{2-\beta}\right) .
$$

(6) gives the series definition for the $E_{q}$-function and shows that it is symmetrical in $\alpha$ and $\beta$.

+ Cf. [3] Evaluating the integral (4) by considering the residues at the poles of $G(\alpha-s)$, we get an expression which is identically equal to the left-hand side. 
3. Recurrence relations. We now prove the following recurrence relations:

$$
\begin{gathered}
\left(1-q^{\alpha}\right) E_{q}(\alpha, \beta:: z)-E_{q}(\alpha+1, \beta:: z)=\frac{q}{z}^{\alpha} E(\alpha+1, \beta+1:: z), \\
\left(q^{\beta}-q^{\alpha}\right) E_{q}(\alpha, \beta:: z)+q^{\alpha} E_{q}(\alpha, \beta+1:: z)=q^{\beta} E_{q}(\alpha+1, \beta:: z), \\
\left(1-q^{\beta}\right) E_{q}(\alpha, \beta:: z)=z^{-1} q^{\beta}\left(1-q^{\alpha-\beta-1}\right) E_{q}(\alpha, \beta+1:: z)+\left(1-q^{\alpha-1}\right) E_{q}(\alpha-1, \beta+1:: z) .
\end{gathered}
$$

To prove (7) we observe that the left-hand side is equal to

$$
\begin{aligned}
& \left(1-q^{\alpha}\right) \frac{G(\alpha)}{1-q} \operatorname{S}_{0}^{1} E_{q}(q \lambda) \lambda^{\beta-1}{ }_{1} \Phi_{0}(\alpha ;-\lambda / z) d(q \lambda)-\frac{G(\alpha+1)}{1-q}{\underset{S}{0}}_{0} E_{q}(q \lambda) \lambda^{\beta-1}{ }_{1} \Phi_{0}(\alpha+1 ;-\lambda / z) d(q \lambda) \\
& =\frac{G(\alpha+1)}{1-q} \mathrm{~S}_{0}^{1} E_{q}(q \lambda) \lambda^{\beta-1}\left[{ }_{1} \Phi_{0}(\alpha ;-\lambda / z)-{ }_{1} \Phi_{0}(\alpha+1 ;-\lambda / z)\right] d(q \lambda) \\
& =\frac{q^{\alpha} G(\alpha+1)}{z(1-q)}{\underset{S}{0}}_{0} E_{q}(q \lambda) \lambda_{1}^{\beta} \Phi_{0}(\alpha+1 ;-\lambda / z) d(q \lambda) \\
& =z^{-1} q^{\alpha} E_{q}(\alpha+1, \beta+1:: z) \text {. }
\end{aligned}
$$

To prove (8), we take (7) and a similar relation with $\alpha$ replaced by $\beta$. Eliminating $E_{q}(\alpha+1, \beta+1:: z)$ between these two relations we get (8).

To prove (9), we multiply (7) by $q^{\beta}-q^{\alpha}$ and (8) by $1-q^{\alpha}$ and subtract. Changing $\alpha$ to $\alpha-1$ in the result so obtained we obtain (9).

4. A generalization of (9). We next prove the following formula :

$$
\sum_{r=0}^{n} \frac{\left(q^{-n}\right)_{r}\left(q^{\alpha-\beta-n}\right)_{r}\left(q^{\alpha-n}\right)_{n}}{\left(q^{\alpha-n}\right)_{r}(q)_{r}}(-z)^{-r} q^{r(\beta+n)} E_{q}(\alpha-n+r, \beta+n:: z)=\left(q^{\beta}\right)_{n} E_{q}(\alpha, \beta:: z) .
$$

To prove (10) we consider its left-hand side and use the contour integral (5) for the $E_{q}$-function in it. This gives

$$
\frac{1}{2 \pi i} \sum_{r=0}^{n} \frac{\left(q^{-n}\right)_{r}\left(q^{\alpha-\beta-n}\right)_{r}\left(q^{\alpha-n}\right)_{n}}{\left(q^{\alpha-n}\right)_{r}(q)_{r}}(-z)^{-r} q^{r(\beta+n)} \int_{C} \frac{G(\alpha-n+r-s) G(\beta+n-s)}{G(1-s)} \frac{\pi z^{s}}{\sin \pi s} d s .
$$

Putting $s=t+r$, and changing the order of integration, which is obviously justified, we get on simplification

$$
\frac{1}{2 \pi i} \int_{C} \frac{G(\alpha-n-t) G(\beta+n-t)}{G(1-t)} \frac{\pi z^{t}}{\sin \pi t}\left(q^{\alpha-n}\right)_{n 3} \Phi_{2}\left[\begin{array}{c}
q^{-n}, q^{\alpha-\beta-n}, q^{t} ; q \\
q^{\alpha-n}, q^{1+t-\beta-n}
\end{array}\right] d t .
$$

Summing the ${ }_{3} \Phi_{2}$ by the basic analogue of Saalschütz's theorem, we get

$$
\frac{\left(q^{\beta}\right)_{n}}{2 \pi i} \int_{C} \frac{G(\alpha-t) G(\beta-t)}{G(1-t)} \frac{\pi z^{t}}{\sin \pi t} d t=\left(q^{\beta}\right)_{n} E_{q}(\alpha, \beta:: z),
$$

which proves (10). For $n=1,(10)$ reduces to (9).

5. An integral representation for $E_{q}(\alpha, \beta:: z)$. We show that $\dagger$

† We write $f([x+h])$ to denote the series $\Sigma a_{r}(x+h)_{r}$, where $f(x)=\sum a_{r} x$. 


$$
E_{q}(\alpha, \beta:: z)=\frac{1}{1-q} \stackrel{1}{S}_{0} E_{q}\left(\alpha, \beta+1:: z /\left[1-q^{\beta} t\right]\right)[1-q t]_{\beta-1} d(t q),
$$

where $\operatorname{Re} \alpha>0, \operatorname{Re} \beta>0$ and $|q|<1$.

Proof. The right-hand integral is given by

$$
\begin{aligned}
& \frac{1}{1-q} \int_{0}^{1} E_{q}\left(\alpha, \beta+1:: z /\left[1-q^{\beta} t\right]\right)[1-q t]_{\beta-1} d(t q) \\
& =\frac{1}{(1-q)^{2}} G(\beta+1){\underset{0}{S}}_{0}^{1}[1-q t]_{\beta-1} d(t q){\underset{0}{1}}_{0} E_{q}(q \lambda) \lambda^{\alpha-1}{ }_{1} \Phi_{0}\left(\beta+1,-\lambda z^{-1}\left[1-q^{\beta} t\right]\right) d(\lambda q) .
\end{aligned}
$$

On changing the order of integration, which is valid for $\operatorname{Re} \alpha>0, \operatorname{Re} \beta>0$ and $\left|\lambda\left(1-q^{\beta} t\right)\right|<|z|$, this becomes

$$
\frac{1}{(1-q)^{2}} G(\beta+1) \operatorname{S}_{0}^{1} E_{q}(q \lambda) \lambda^{\alpha-1} d(\lambda q){\underset{0}{1}}_{0}^{1}[1-q t]_{\beta-1} \Phi_{0}\left(\beta+1, \lambda z^{-1}\left[1-q^{\beta} t\right]\right) d(t q) .
$$

Expanding the ${ }_{1} \Phi_{0}$ and integrating term by term the $t$-integral, for $\operatorname{Re} \beta>0$, with the help of the result

$$
\frac{1}{(1-q)} \int_{0}^{1} x^{\alpha-1}(1-q x)_{\lambda-1} d(q x)=\prod_{n=0}^{\infty} \frac{\left(1-q^{\alpha+\lambda+n}\right)\left(1-q^{1+n}\right)}{\left(1-q^{\alpha+n}\right)\left(1-q^{\lambda+n}\right)} \quad(\operatorname{Re} \alpha>0, \operatorname{Re} \lambda>0),
$$

we get

$$
\frac{1}{1-q} G(\beta) \stackrel{S}{0}_{0}^{1} E_{q}(\lambda q) \lambda^{\alpha-1}{ }_{1} \Phi_{0}(\beta ;-\lambda / z) d(q \lambda)=E_{q}(\alpha, \beta:: z) .
$$

6. An asymptotic expansion for $E_{q}(\alpha, \beta:: z)$ for $|z| \rightarrow \infty$. If we evaluate the integral (5) by considering the residues at the poles of $\Gamma(s)$, we deduce the behaviour of $E_{q}(\alpha, \beta:: z)$ for large values of $|z|$. In particular, we find that

$$
E_{q}(\alpha, \beta:: z) \sim \frac{G(\alpha) G(\beta)}{G(1)}{ }_{2} \Phi_{0}(\alpha, \beta ;-1 / z)
$$

7. It may be of interest to generalize the $E_{q}(\alpha, \beta:: z)$ function and also to define the basic analogues of the Whittaker functions $W_{k, m}$ and $M_{k, m}$ with its help, as in the case of MacRobert's function, and then to study further properties of such functions. I hope to deal with these functions in a subsequent paper.

\section{REFERENCES}

1. W. Hahn, Über die höheren Heineschen Reihen und eine einheitliche Theorie der sogenannten speziellen Funktionen, Math. Nachr., 3 (1950), 257-294.

2. T. M. MacRobert, Induction proofs of the relations between certain asymptotic expansions and corresponding generalised hypergeometric series, Proc. Roy. Soc. Edinburgh 58 (1937), 1-13.

3. G. N. Watson, The continuations of functions defined by generalised hypergeometric series, Trans. Cambridge Phil. Soc. 21 (1909), 281-299.

\section{LUCKNOW UNIVERSITY}

INDIA 